\title{
Recuperación de suelos contaminados por elementos traza mediante fitotecnologías de estabilización
}

\author{
T. Pardo ${ }^{1, *}$ \\ (1) Departamento de Conservación de Suelos y Agua y Manejo de Residuos Orgánicos, CEBAS-CSIC, Apdo. de correos 164, 30100 Espinardo, Murcia, \\ España.
}

*Autor de correpondencia: T. Pardo [tpardo@cebas.csic.es; tpardoiglesias@gmail.com]

> Recibido el 9 de agosto de 2012, aceptado el 14 de septiembre de 2013.

Pardo, T. (2013). Recuperación de suelos contaminados por elementos traza mediante fitotecnologías de estabilización. Ecosistemas 22(2):122-124. Doi.: 10.7818/ECOS.2013.22-2.19

La contaminación de suelos por elementos traza debida a la actividad minera generalmente conlleva la degradación y modificación de su capacidad para desarrollar plenamente y de manera sostenible sus funciones ecológicas potenciales, pudiendo además afectar a los ecosistemas adyacentes. Ante esta problemática surge la necesidad de desarrollar técnicas que puedan reducir la toxicidad de los elementos traza tratándolos y estabilizándolos in situ, y que a su vez fomenten los procesos naturales que tienen lugar en el suelo con objeto de recuperar su funcionalidad (o salud). En este sentido, una de las técnicas medioambientalmente más respetuosas y económicamente viables para la recuperación de zonas mineras es la fitoestabilización. Esta fitotecnología se basa en el uso de plantas tolerantes y prácticas agronómicas para disminuir la disponibilidad de los contaminantes mediante la mejora de las condiciones fisicoquímicas y biológicas del suelo y su protección física frente a la erosión y a la lixiviación (Wenzel et al. 1999). Esta es una de las técnicas que menos riesgos asociados implica para los demás compartimentos naturales (Vangrosveld et al. 2009; Mench et al. 2010).

En este marco se planteó la presente investigación, cuyo objetivo principal fue estudiar, en suelos mineros contaminados por elementos traza, el efecto de la aplicación de técnicas de fitoestabilización basadas en el uso de enmiendas orgánicas y plantas autóctonas, sobre las propiedades que determinan la salud del suelo. Todo ello con el fin de establecer las bases para evaluar su eficacia y optimizar su aplicación, y a su vez, potenciar los beneficios ambientales y socioeconómicos relacionados con la utilización de residuos orgánicos con gran problemática de gestión como enmiendas del suelo para mejorar su fertilidad.

Así, se desarrollaron una serie de ensayos bajo tres escalas o niveles de experimentación: laboratorio, cámara de cultivo y campo, utilizando tres suelos procedentes de la Sierra Minera de La UniónCartagena (Murcia) con diferentes características fisicoquímicas y grado de contaminación en cuanto a la concentración de elementos traza (total y disponible) y $\mathrm{pH}$ (moderado, alto y extremo, Fig.1 y Tabla 1). Como enmiendas orgánicas se utilizaron dos materiales con diferente grado de estabilidad microbiológica: compost de alperujo y purín de cerdo. El primero procede de la transformación mediante compostaje del principal residuo de la industria olivarera

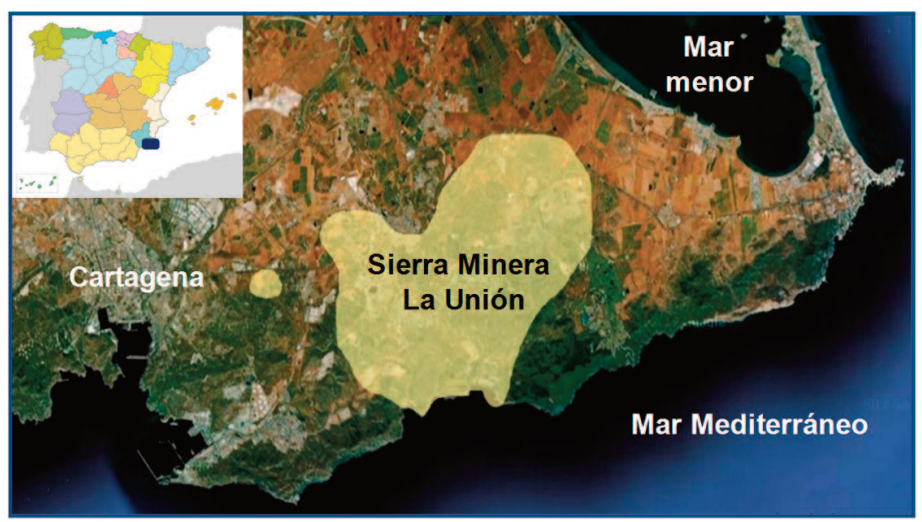

Figura 1. Localización de la Sierra Minera de La Unión-Cartagena

Tabla 1. Caracterización de los suelos utilizados en los distintos experimentos. SG, LL1 y LL2: suelos de contaminación moderada, alta y extrema, respectivamente. Las concentraciones de elementos traza corresponden a contenido total en el suelo

\begin{tabular}{lccc}
\hline Parámetro & SG & LL1 & LL2 \\
\hline $\mathrm{pH}$ & 7.6 & 6.2 & 3.5 \\
$\mathrm{CaCO}_{3}$ & 19.5 & 2.1 & 0.7 \\
$\mathrm{CE}\left(\mathrm{dS} \mathrm{m}^{-1}\right)$ & 0.2 & 2.7 & 4.2 \\
$\mathrm{C}_{\mathrm{OT}}\left(\mathrm{g} \mathrm{kg}^{-1}\right)$ & 22.5 & 1.3 & 1.3 \\
$\mathrm{~N}_{\mathrm{T}}\left(\mathrm{g} \mathrm{kg}^{-1}\right)$ & 0.9 & 0.4 & 0.4 \\
$\mathrm{As}$ & $65\left(\mathrm{mg} \mathrm{kg}^{-1}\right)$ & 664 & 712 \\
$\mathrm{Cd}$ & $\left(\mathrm{mg} \mathrm{kg}^{-1}\right)$ & 19 & 6 \\
$\mathrm{Cu}$ & $\left(\mathrm{mg} \mathrm{kg}^{-1}\right)$ & 193 & 147 \\
$\mathrm{Fe}_{\mathrm{T}}\left(\mathrm{g} \mathrm{kg}^{-1}\right)$ & 36 & 108 & 122 \\
$\mathrm{Mn}_{\mathrm{T}}\left(\mathrm{g} \mathrm{kg}^{-1}\right)$ & 6.80 & 4.07 & 0.98 \\
$\mathrm{~Pb}_{\mathrm{T}}\left(\mathrm{mg} \mathrm{kg}^{-1}\right)$ & 651 & 10188 & 14532 \\
$\mathrm{Zn}$ & $632\left(\mathrm{mg} \mathrm{kg}^{-1}\right)$ & 9686 & 3873 \\
\hline
\end{tabular}


(alperujo), y el segundo es el principal residuo de la ganadería porcina, dos de los sectores agropecuarios más importantes de España. Además, se utilizaron cal hidratada y un fertilizante inorgánico NPK como enmiendas inorgánicas. En cuanto a las especies vegetales, se utilizó Lolium perenne L. en el ensayo realizado en cámara de cultivo para actuar como indicador de la disponibilidad y fitotoxicidad de los elementos traza, la cual se plantó en todos los tratamientos excepto en suelo sin enmendar para controlar el efecto de las plantas. Además se utilizaron Atriplex halimus L. y Bituminaria bituminosa (L.) C.H. Stirton en los ensayos en campo por ser especies tolerantes a los elementos traza y autóctonas de la zona de estudio, estando así adaptadas a las condiciones edafoclimáticas de la misma. En dichos ensayos ambas especies se plantaron solas o en combinación (según el suelo), manteniendo parcelas sin plantar en cada tratamiento como control del efecto planta.

El estudio de los distintos tratamientos bajo las tres perspectivas experimentales (Fig. 2), se planteó como una estrategia apropiada para evaluar su influencia sobre las propiedades fisicoquímicas y biológicas del suelo que definen la salud del mismo, cuya mejora es en definitiva el fin último de la fitoestabilización (Epelde et al. 2009). De este modo, los experimentos permitieron estudiar a corto, medio y largo plazo, bajo condiciones controladas y naturales, (i) los efectos de las enmiendas orgánicas e inorgánicas sobre la movilidad, disponibilidad y toxicidad de los elementos traza, y (ii) su influencia en el desarrollo vegetal y microbiano. Lo cual se realizó distinguiendo entre el efecto de la propia materia orgánica aportada por las enmiendas orgánicas (compost y purín) y la modificación de las características fisicoquímicas producida tras su aplicación, gracias a la comparación con los materiales inorgánicos (cal o fertilizante). Asimismo, estos ensayos permitieron evaluar la adecuación de las especies vegetales seleccionadas y estudiar la importante interrelación existente entre las plantas y las comunidades microbianas del suelo.

En los distintos ensayos se observó que la solubilidad y movilidad de los elementos traza en los suelos estudiados dependió principalmente del $\mathrm{pH}$ y de las condiciones redox del suelo, por lo que el efecto de las enmiendas sobre su disponibilidad estuvo asociado fundamentalmente a la modificación de estos parámetros, no siendo tan importante la naturaleza de la materia orgánica aportada. De este modo, el efecto de las enmiendas sobre los elementos traza en los suelos con contaminación moderada y alta (con pH cercanos a la neutralidad) fue escaso, destacando únicamente una leve solubilización de As y Mn, y cierta inmovilización de $\mathrm{Pb}$ y Zn. Sin embargo, en el suelo con contaminación extrema, de mayor acidez, el uso de las enmiendas orgánicas implicó una importante inmovilización de todos los elementos traza estudiados (excepto el arsénico) gracias al incremento del $\mathrm{pH}$ y a la modificación de las condiciones redox (Tabla 2).

Los suelos con mayor nivel de contaminación (alta y extrema) produjeron una gran toxicidad para plantas, invertebrados y microrganismos, tal y como se observó en los tests de ecotoxicidad realizados, tanto directos (con Zea mays, Lactuca sativa, Eisenia fetida) como indirectos (con Lactuca sativa, Lepidium sativum, Daphnia magna, Thamnocephalus platyurus y Vibrio fisheri). Dicha

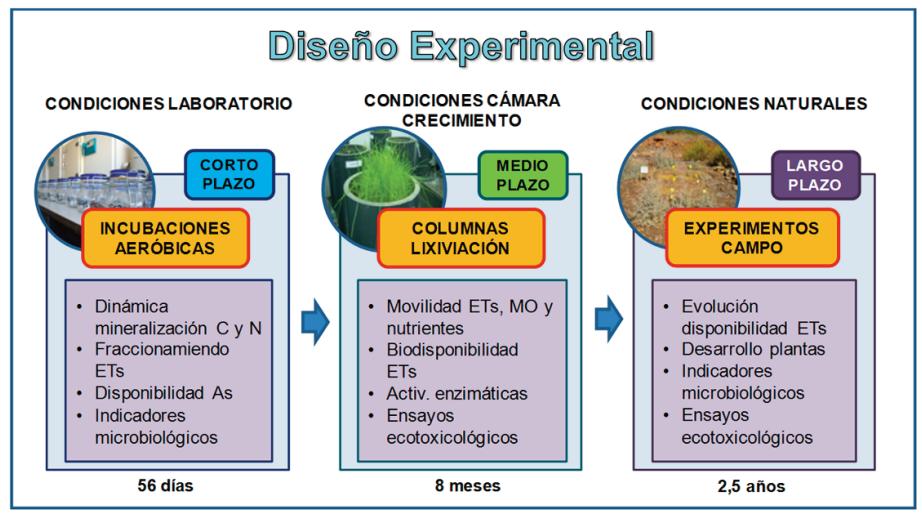

Figura 2. Diseño experimental de la presente Tesis doctoral. ETs: elementos traza; MO: materia orgánica.

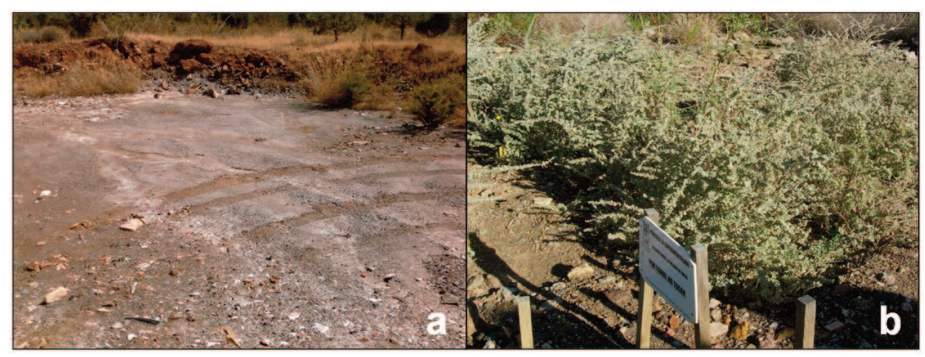

Figura 3. Evolución del proceso de fitoestabilización del suelo LL1 (altamente contaminado). Estado inicial de la zona de actuación (a), zona tras 2.5 años de fitoestabilización (b).

toxicidad fue debida principalmente a la salinidad, la acidez y la disponibilidad de los elementos traza de ambos suelos, pudiendo considerarse que su función "hábitat" y su función "retención" se encontraban afectadas. Pero la adición de las enmiendas orgánicas a estos suelos logró disminuir considerablemente su toxicidad y con ello su posible impacto sobre el medio ambiente.

La adición de los materiales orgánicos supuso una importante fuente de carbono orgánico y nutrientes esenciales $(\mathrm{N}, \mathrm{P}, \mathrm{K})$, cuya disponibilidad y persistencia en el tiempo en todos los suelos (monitorizada periódicamente en los distintos experimentos) dependió de la naturaleza de las enmiendas y de la complejidad de la materia orgánica aportada. El compost, debido a la estabilidad de su materia orgánica y su lenta mineralización, significó una reserva de nutrientes a largo plazo (todavía disponibles transcurridos 2.5 años desde su aplicación), mientras que la naturaleza lábil del purín implicó la disponibilidad a corto plazo (meses) de los mismos. Este aporte de nutrientes esenciales por parte del compost y del purín, pese a su diferente persistencia y a la importante toxicidad observada en los suelos, mejoró el crecimiento vegetal, tanto de las especies seleccionadas (especialmente de A. halimus, Fig. 3), como de especies espontáneas, fomentando así el establecimiento de una cubierta vegetal bien desarrollada, permanente y autosostenible en el suelo (las plantas produjeron frutos con semilla fértil). Esto supuso una fuente extra de nutrientes como consecuencia de la

Tabla 2. Concentración de elementos traza extraíbles con CaCl2 ( $\left.\mathrm{mg} \mathrm{kg}^{-1}\right)$ en el suelo de la capa superficial de las columnas (0-10 cm) tras 8 meses de experimentación (experimento en cámara de cultivo).

\begin{tabular}{|c|c|c|c|c|c|}
\hline Tratamiento & Al & As & Mn & $\mathrm{Pb}$ & $\mathrm{Zn}$ \\
\hline Control-sin plantar & $385 \pm 37 a$ & $0.19 \pm 0.03 b$ & $487 \pm 48 a$ & $307 \pm 23 a$ & $1611 \pm 132 a$ \\
\hline Control & $297 \pm 17 b$ & $0.17 \pm 0.01 b$ & $268 \pm 46 b$ & $305 \pm 43 a$ & $991 \pm 159 b$ \\
\hline Cal & $9.6 \pm 5.0 \mathrm{c}$ & $0.64 \pm 0.05 a$ & $281 \pm 34 b$ & $38 \pm 10 b$ & $1082 \pm 93 b$ \\
\hline Compost & $0.1 \pm 0.3 c$ & $0.66 \pm 0.02 a$ & $320 \pm 8 b$ & $13 \pm 2 b$ & $1155 \pm 44 b$ \\
\hline Purín & $10.6 \pm 5.8 c$ & $0.52 \pm 0.07 a$ & $246 \pm 29 b$ & $52 \pm 14 b$ & $1009 \pm 50 b$ \\
\hline ANOVA & $* * *$ & $* * *$ & $* * *$ & $* * *$ & ** \\
\hline
\end{tabular}




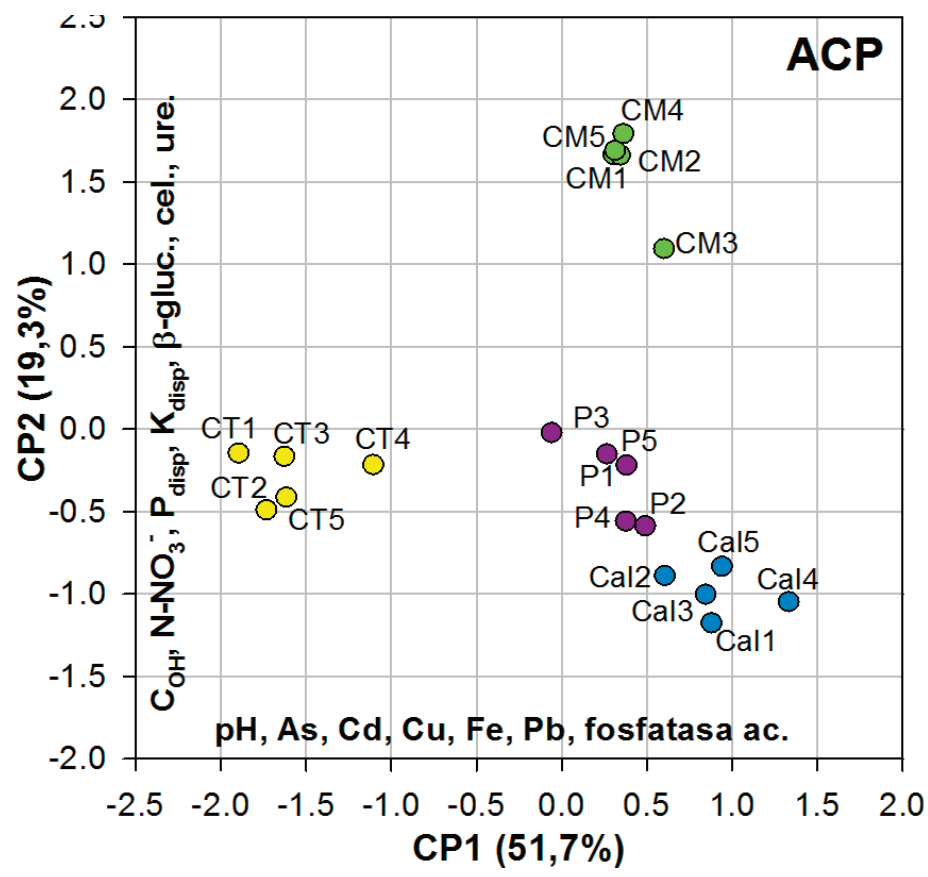

Figura 4. Integración de las propiedades fisicoquímicas y microbiológicas de la capa superficial de las columnas (suelo de contaminación extrema) mediante análisis por componentes principales (ACP). CT: suelo sin enmienda; Cal: suelo tratado con cal hidratada; CM: suelo tratado con compost; P: suelo tratado con purín (todos con Lolium perenne). Сон: C orgánico hidrosoluble; $P_{\text {disp: }} P$ disponible; $K_{\text {disp: }}$ K disponible; $\beta$-gluc.: $\beta$-glucosidasa; cel.: celulasa; ure: ureasa; fosfatasa ac.: fosfatasa ácida.

degradación del material vegetal incorporado al suelo durante el ciclo de vida de las plantas, pudiendo suplir la menor persistencia de dichos elementos en el tratamiento con purín. Todo ello estimuló el desarrollo microbiano en los distintos suelos al reducir las condiciones de estrés, aumentando a corto (meses) y largo plazo (años) su crecimiento, actividad y complejidad funcional (Fig. 4), lo cual implicó la reactivación de procesos esenciales que se encontraban inhibidos, como la nitrificación, que a su vez mejoró el crecimiento vegetal.

La integración de todos los resultados obtenidos respecto al suelo, planta y disolución del suelo indica que la adición de los materiales orgánicos supuso la mejora de la función hábitat del ecosistema edáfico, la reactivación de los ciclos biogeoquímicos de los principales elementos y la reducción de la dispersión de los ele- mentos traza a los ecosistemas adyacentes, mejorando por tanto, la salud y funcionalidad de los suelos (Gomez-Sagasti 2012), y lo más importante, todo ello pudo ser verificado en campo bajo condiciones reales. Sin embargo, la adición de cal hidratada o de fertilizante inorgánico no logró mejorar significativamente estos parámetros pese incrementar el $\mathrm{pH}$ del suelo y aportar nutrientes fácilmente asimilables, respectivamente, reflejando la necesidad del aporte de la materia orgánica y de su persistencia en el suelo.

Por tanto, la utilización tanto de compost de alperujo como de purín de cerdo, y especialmente en combinación con $A$. halimus, puede ser una estrategia eficaz para la recuperación de la salud de los suelos pobres con elevados niveles de elementos traza propios de la Sierra Minera de La Unión, siendo esta fitotecnología potencialmente aplicable a gran escala y extrapolable a suelos de zonas climáticas semiáridas con similares características. Además, estos resultados muestran la idoneidad de la utilización de materiales orgánicos como enmiendas en este tipo de técnicas, lo cual además supone una alternativa medioambientalmente respetuosa para su reciclado. Luego, teniendo en cuenta la gran problemática de gestión que existe en España asociada a dichos residuos, su utilización puede ser muy interesante, suponiendo importantes beneficios ambientales y socioeconómicos.

\section{Referencias}

Epelde, L., Becerril, J.M., Mijangos, M., Garbisu, C. 2009. Evaluation of the efficiency of a phytostabilizations process with biological indicators of soil health. Journal of Environmental Quality 38:2041-2049.

Gómez-Sagasti, M.T.., Alkorta, I., Becerril, J.M., Epelde, L., Anza, M., Garbisu, C. 2012. Microbial Monitoring of the Recovery of Soil Quality During Heavy Metal Phytoremediation. Water, Air, and Soil Pollution 223:3249-3262.

Mench, M., Lepp, N., Bert, V., Schwitzguébel, J.P., Gawronski, S.W., Schröder, P., Vangronsveld, J. 2010. Successes and limitations of phytotechnologies at field scale: outcomes, assessment and outlook from COST Action 859 Journal Soils Sediments 10:1039-1070

Vangronsveld, J., Herzig, R., Weyens, N., Boulet, J., Adriaensen, K., Ruttens, A., Thewys, T., Vassilev, A., Meers, E., Nehnevajova, E., Van der Lelie, D., Mench, M. 2009. Phytoremediation of contaminated soils and groundwater: lessons from the field. Environmental Science and Pollution Research 16:765-794.

Wenzel, W.W., Salt, D., Smith, R., Adriano, D.C. 1999. Phytoremediation: a plant microbe based remediation system. En: Adriano, D.C., Bollag, J.M., Frankenberger Jr., W.T., Sims, R.C. (Eds.). Bioremediation of Contaminated Soils, Agronomy Monograph No. 37, pp. 456-508. Soil Science Society of America, Madison, WI, USA.

\section{TANIA PARDO}

\section{Recuperación de suelos contaminados por elementos traza mediante fitotecnologías de estabilización}

\section{Tesis Doctoral (con Mención Europea)}

Departamento de Conservación de Suelos y Agua y Manejo de Residuos Orgánicos, CEBAS-CSIC. Departamento de Biología Animal, Ecología, Parasitología, Edafología y Química Agrícola, Universidad de Salamanca.

1 de Marzo 2013

Directores: Dra. Ma Pilar Bernal Calderón y Dr. Rafael Clemente Carrillo

\section{Publicaciones resultantes de la tesis}

Alburquerque, J.A., Clemente, R., de la Fuente, C., Martínez-Alcalá, I. Pardo, T., Bernal, M.P. 2010. El compost de alperujo como enmienda orgánica en estrategias de recuperación de suelos contaminados por metales pesados. Boletín de compostaje para producción ecológica (Consejería de Agricultura y Pesca, Junta de Andalucía). $4^{\circ}$ trimestre, 5-6.

Bernal, M.P., de la Fuente, C., Alburquerque, J.A., Martínez-Alcalá, I., Pardo, T., Clemente, R. 2009. Opciones de recuperación de suelos del entorno del Mar Menor afectados por la actividad minera. En: El Mar Menor, estado actual del conocimiento científico. pp 399-449, Fundación IEA, Murcia, España.

Bernal, M.P., Clemente, R., de la Fuente, C., Pardo, T., Alburquerque, J.A., Bustamante, M.A., Martínez-Alcalá, I., Martínez-Fernández, D.,
Walker D.J. 2010. Nuevas opciones de revalorización del purín de porcino. Tierras de Castilla y León: Ganadería 174:54-57.

Clemente, R., Walker, D.J., Pardo, T., Fernández, D., Bernal, M.P. 2012. The use of a halophytic plant species and organic amendments for the remediation of a trace elements-contaminated soil under semi-arid conditions. 2012. Journal of Hazardous Materials, 223:63-71.

Pardo, T., Martínez-Fernández, D., Clemente, R., Bernal, M.P., Walker, D.J. 2013. Native plants and organic amendment to promote the vegetation cover in a trace elements-contaminated soil. Environmental Science and Pollution Research. Doi.: 10.1007/s11356-013-1988-z

Pardo, T., Clemente, R., Bernal. M.P. 2011. Effects of compost, pig slurry and lime on trace element solubility and toxicity in two soils differently affected by mining activities. Chemosphere 84:642-650. 